\title{
Construction of microRNA and transcription factor regulatory network based on gene expression data in cardiomyopathy
}

\author{
Lei Wang ${ }^{*}$, Jihua Hu, Haijian Xing, Min Sun, Juanli Wang, Qiang Jian and Hua Yang
}

\begin{abstract}
Background: Cardiomyopathy is a progressive myocardial disorder. Here, we attempted to reveal the possible mechanism of cardiomyopathy at the transcription level with the roles of microRNAs (miRNAs) and transcription factors (TFs) taken into account.

Method: We firstly identified differentially expressed genes (DEGs) between cardiomyopathy patients and controls with data from the gene expression omnibus (GEO) database. DEGs were associated with the canonical pathways, molecular and cellular functions, physiological system development and function in the Ingenuity Knowledge Base by using the Ingenuity Pathway Analysis (IPA) software. TFs and miRNAs that DEGs significantly enriched were identified and a double-factor regulatory network was constructed.

Results: A total of 1,680 DEGs were identified. The DEGs were enriched for various pathways, with glucocorticoid receptor signaling as the most significant. A double-factor regulatory network was constructed, including seven TFs and two miRNAs. A subnetwork under the regulation of MEF2C and SRF was also constructed to illustrate their regulatory effects on cardiac functions.
\end{abstract}

Conclusion: Our results may provide new understanding of cardiomyopathy and may facilitate further therapeutic studies.

Keywords: Cardiomyopathy, Transcription factors, miRNAs, Gene expression

\section{Background}

Cardiomyopathy is a progressive myocardial disorder, usually leading to cardiovascular death or heart failure-related disability [1]. It develops at any age, in either sex, and in any population $[2,3]$. The etiology of cardiomyopathy is highly complex and improving its treatments has become a research hotspot.

Using bioinformatics combined with gene expression data to identify potential therapeutic targets has shown great application prospects. The majority of the previous studies mainly focused on the analyses of differentially expressed genes (DEGs), without considering microRNAs (miRNAs) and transcription factors (TFs) that regulate the expression of DEGs. miRNAs are small non-coding RNAs that control various biological processes through affecting

\footnotetext{
* Correspondence: xawanglei@163.com

Department of Cardiology, Xi'an Children's Hospital, 69 Xi Ju Rd, Lianhu District, Xi'an 710003, China
}

the stability and translation of target mRNAs. Previous studies have proposed several miRNAs as being involved in the pathogenesis of cardiomyopathy, such as miR-1 [4] and miR-21 [5]. TFs can regulate gene expression through binding to the cis-elements in target genes' promoter regions. TFs, such as $M E F 2$, have been reported to be associated with cardiomyopathy [6]. Considering the important regulatory roles of miRNAs and TFs in the pathogenesis of cardiomyopathy, identification of miRNAs and TFs that enriched with target DEGs and construction of a double-factor regulatory network may provide new understanding of the molecular mechanism of cardiomyopathy.

In the current study, based on gene expression data from the Gene Expression Omnibus (GEO) database, we acquired DEGs, miRNAs and TFs that enriched with target DEGs and constructed a double-factor regulatory network. 
Our results may reveal the possible mechanism of cardiomyopathy at the transcription level.

\section{Methods}

\section{Ethics Statement}

This study was approved by the institutional review board of the Xi'an Children's Hospital (20140518).
Written informed consent was obtained from all patients for the publication of this report and any accompanying images.

\section{Microarray data}

The gene expression profile GSE5406 from the GEO database was used. This dataset includes transcription

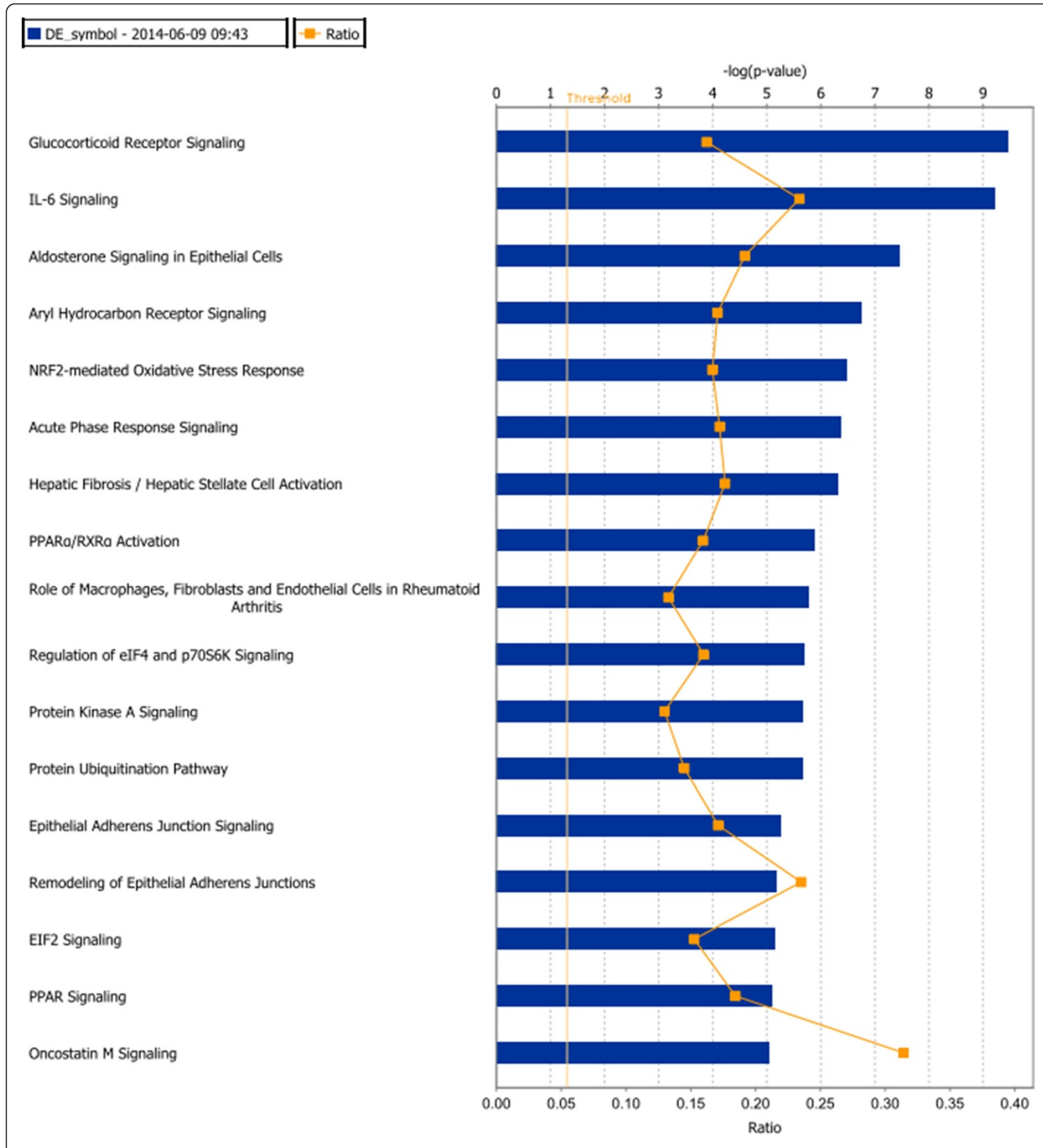

Figure 1 Ingenuity Pathway Analysis results. The significant canonical pathways in which differently expressed genes (DEGs) were enriched are shown. 
profiles of 210 left ventricular myocardial tissue samples, 194 of which were from patients with advanced cardiomyopathy and 16 of which were from healthy donors. All patients had New York Heart Association class 3 to 4 symptoms and left ventricular systolic dysfunction, with ejection fraction of $14 \pm 8 \%$ (mean \pm SD). Patients suffered from heart failure due to ischemic $(\mathrm{n}=86)$ or idiopathic dilated ( $\mathrm{n}=108)$ cardiomyopathy. Control samples had normal left ventricular function with ejection fraction of $56 \pm 7 \%$ ( $P=0.0001$ versus patients). None of the subjects received mechanical support with left ventricular assist devices. Myocardial tissue samples were obtained from patients undergoing heart transplantation and from controls deemed unsuitable for transplantation. Whole hearts were removed after preservation in cold cardioplegia at the time of transplantation or donor harvest. Then, segments of noninfarcted left ventricular free wall were snap-frozen in liquid nitrogen. For each sample, RNA was isolated by using Trizol reagent (Invitrogen, Carlsbad, CA, USA). The dataset was generated by using the (HG-U133A) Affymetrix Human Genome U133A Array (Affymetrix, Santa Clara, CA, USA).

\section{Identification of differentially expressed genes (DEGs)}

Raw data from all arrays were normalized using Robust Multi-array Analysis (RMA) [7] in the R software (version 3.0.0). The resulting expression values were used to identify DEGs with the limma package (3.12.1) in R. DEGs were detected by using $t$-tests and multiple test corrections were carried out with the Benjamini-Hochberg method [8]. The threshold for significance was set as $P<0.01$.

\section{Enrichment analysis}

To explore the functions and pathways of DEGs, DEGs were associated with the canonical pathways, molecular and cellular functions, physiological system development and function in the Ingenuity Knowledge Base by using the Ingenuity Pathway Analysis (IPA) software (Ingenuity ${ }^{\circ}$ Systems, http://www.ingenuity.com).

\section{Construction of miRNA-TF regulatory network}

We also acquired miRNAs and TFs which were overrepresented with target DEGs. For miRNA analysis, we used Targetscan [9], miRanda [10] and Pita [11] for miRNA target DEGs prediction. To avoid false positive results, miRNA-DEG prediction results supported by all three prediction methods were considered to be confidential. For TF analysis, we obtained TF binding sites and the coordinate's position information of human being from the University of California Santa Cruz (UCSC) database. TFs and miRNAs that DEGs significantly enriched were identified by using the hypergeometric distribution test. $P<0.01$ was set as the threshold. Based on the regulatory relationship between miRNAs or TFs and DEGs, a network was constructed by using the IPA software.

\section{Results}

A total of 1,680 DEGs were identified, including 963 down-regulated genes and 717 up-regulated ones in advanced cardiomyopathy patients.

As shown in Figure 1, the IPA results of canonical pathways showed that the DEGs were enriched for various pathways, among which glucocorticoid receptor signaling was the most significant. Molecular and cellular functions analysis revealed that 'Cell Death and Survival' $\left(P=2.53 \times 10^{-24}\right.$ to $\left.1.36 \times 10^{-3}\right)$ was the top molecular function affected by DEGs followed by 'Cellular Growth and Proliferation' $\left(P=3.37 \times 10^{-24}\right.$ to $1.36 \times 10^{-3}$ ) (Table 1). 'Cardiovascular System Development and Function' $\left(P=4.17 \times 10^{-12}\right.$ to $\left.1.20 \times 10^{-3}\right)$ was the top physiological function mediated by DEGs (Table 1).

Table 1 Ingenuity Pathway Analysis: functions related to differentially expressed genes

\begin{tabular}{lll}
\hline Name & P-value & Number of molecules \\
\hline Molecular and Cellular Functions & & 405 \\
Cell Death and Survival & $2.53 \times 10^{-24}$ to $1.36 \times 10^{-3}$ & 414 \\
Cellular Growth and Proliferation & $3.37 \times 10^{-24}$ to $1.36 \times 10^{-3}$ & 236 \\
Cellular Movement & $5.41 \times 10^{-13}$ to $1.36 \times 10^{-3}$ & 247 \\
Gene Expression & $8.14 \times 10^{-11}$ to $1.20 \times 10^{-3}$ & 328 \\
Cellular Development & $1.13 \times 10^{-10}$ to $1.36 \times 10^{-3}$ & 125 \\
Physiological System Development and Function & & 92 \\
Cardiovascular System Development and Function & $4.17 \times 10^{-12}$ to $1.20 \times 10^{-3}$ & 163 \\
Organismal Development & $3.96 \times 10^{-11}$ to $1.22 \times 10^{-3}$ & 117 \\
Tissue Development & $3.68 \times 10^{-8}$ to $1.22 \times 10^{-3}$ & $3.84 \times 10^{-7}$ to $1.20 \times 10^{-3}$ \\
Tumor Morphology & $8.52 \times 10^{-6}$ to $1.24 \times 10^{-3}$ & 40 \\
Connective Tissue Development and Function & & \\
\hline
\end{tabular}


Table 2 Transcription factors and microRNAs (miRNAs) enriched with target differentially expressed genes (DEGs)

\begin{tabular}{ll}
\hline Name & $P$-value \\
\hline MicroRNA & \\
miR-30c-5p (and other miRNAs w/seed GUAAACA) & $3.12 \times 10^{-4}$ \\
miR-125b-5p (and other miRNAs w/seed CCCUGAG) & $2.15 \times 10^{-3}$ \\
Transcription factors & \\
MEF2C & $5.65 \times 10^{-6}$ \\
GATA1 & $6.11 \times 10^{-5}$ \\
SRF & $1.53 \times 10^{-4}$ \\
NUPR1 & $4.18 \times 10^{-4}$ \\
ATF4 & $7.25 \times 10^{-4}$ \\
RELA & $9.35 \times 10^{-3}$ \\
GLI2 & $1.42 \times 10^{-2}$ \\
\hline
\end{tabular}

The results of miRNA and TF enrichment analysis are listed in Table 2. A network was constructed to illustrate the regulatory relationships (Figure 2). Considering the important role of $S R F$ and $M E F 2 C$ in the progression of cardiomyopathy, a subnetwork under their regulation was constructed and the biological functions they may affect were also indicated (Figure 3).

\section{Discussion}

In the current study, based on GSE5406 from the GEO database, 1,680 DEGs were identified between advanced cardiomyopathy patients and healthy controls. DEGs were mapped into the Ingenuity Knowledge Base and several canonical pathways were screened out, with glucocorticoid receptor signaling as the most significant. Glucocorticoid receptors are involved in cardiovascular homeostasis [12] and glucocorticoid could protect rodent hearts from ischemia/reperfusion injury [13]. Our results confirmed the essential roles of this pathway in the pathogenesis of cardiomyopathy. Molecular and cellular functions analysis revealed

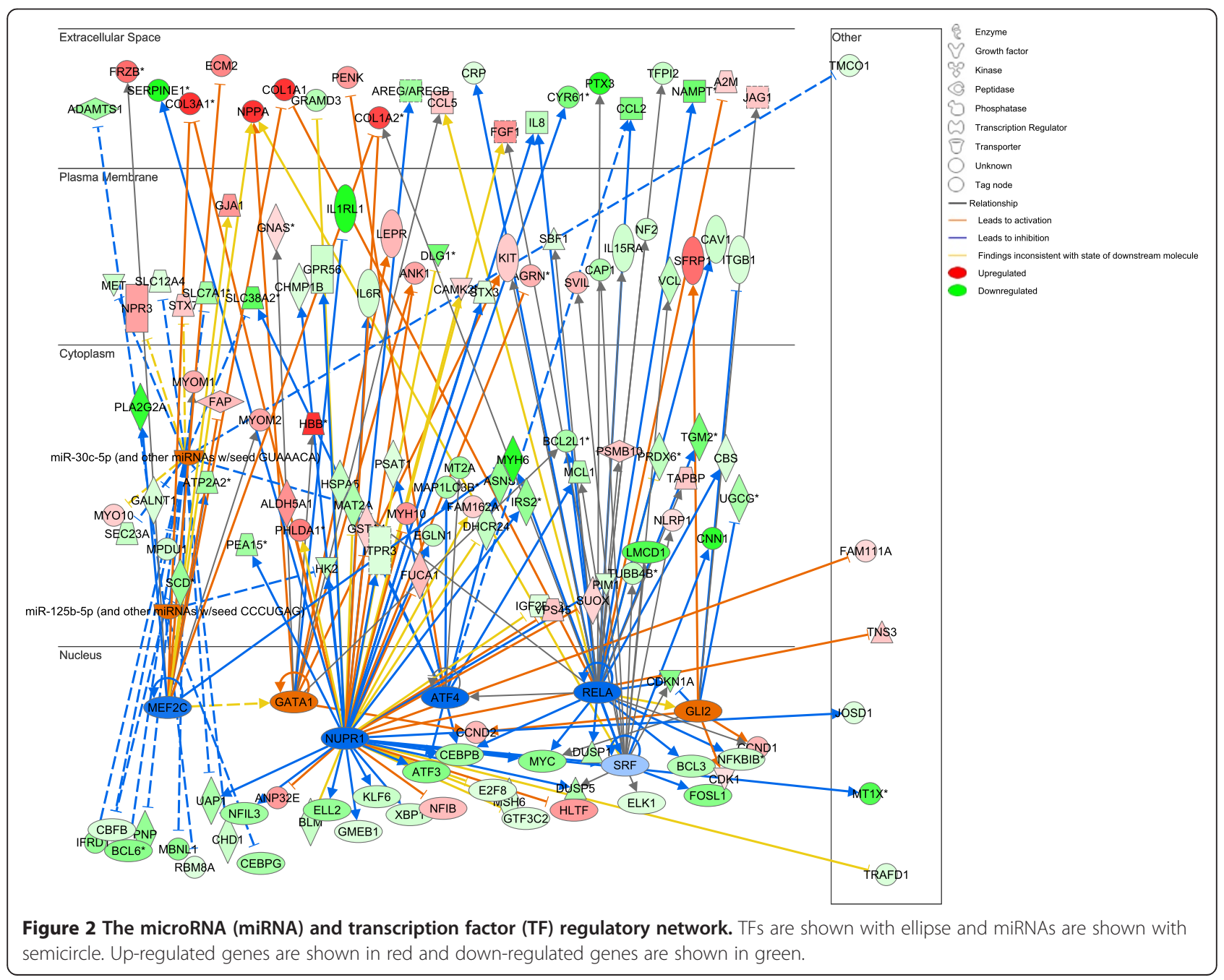




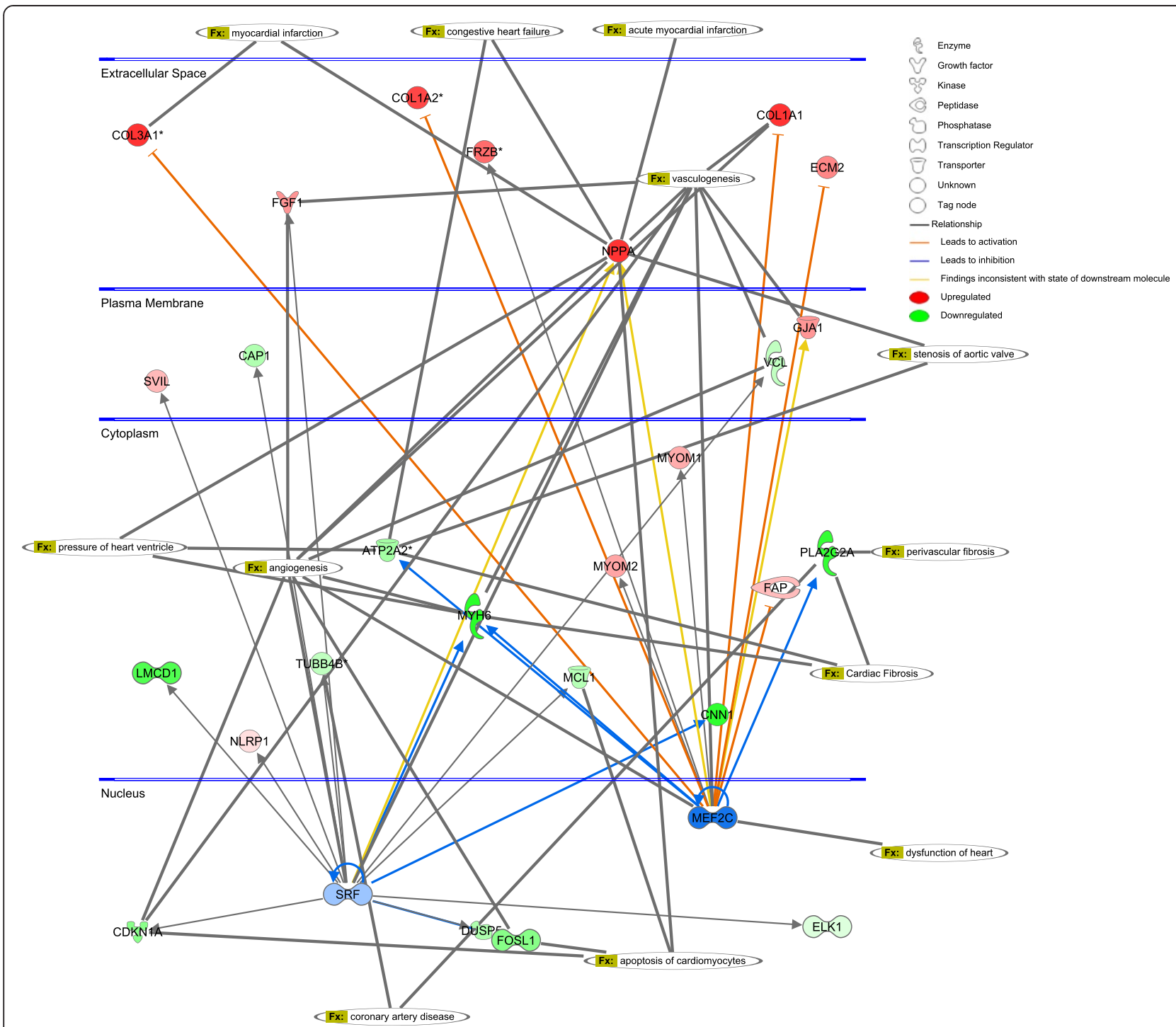

Figure 3 The network under the regulation of SRF and MEF2C. Up-regulated genes are shown in red and down-regulated genes are shown in green. The related cardiac functions are indicated in long ellipses.

that 'Cell Death and Survival' $\left(P=2.53 \times 10^{-24}\right.$ to $\left.1.36 \times 10^{-3}\right)$ was the top molecular function affected by DEGs, suggesting that dysregulation of the cell function may contribute to the progressive heart failure induced by cardiomyopathy.

TFs or miRNAs that DEGs significantly enriched were identified and a double-factor regulatory network was constructed, including seven TFs and two miRNAs (Figure 2). All these miRNAs and TFs have previously been implicated in cardiomyogenesis or cardiac function. Considering their important regulatory roles in controlling various biological processes, these miRNAs and TFs may provide new avenues for the therapeutic strategies of cardiomyopathy. In addition, since all these miRNAs and TFs were detected in patients suffered from New York Heart Association class 3 to 4 symptoms, they may also be considered as prognosis markers in clinical practice. A subnetwork under the regulation of $S R F$ and $M E F 2 C$ was also constructed and the functions they may affect were also indicated (Figure 3) considering the considerable evidence in support of their involvement in cardiomyopathy [14-17]. As shown in Figure 3, both $S R F$ and MEF2C may inhibit the expression of MYH6 and CNN1. MYH6 is a famous cardiac muscle myosin gene and mutations of this gene are implicated in cardiomyopathy in various studies [18]. Inhibition of CNN1 expression may disrupt its suppression of cardiomyopathy through the $\varepsilon P K C$ pathway [19]. In addition, both SRF and $M E F 2 C$ may regulate the expression of NPPA, which was overexpressed in patients in our results. This gene is involved in many cardiac dysfunctions (Figure 3), including myocardial infarction, heart failure, and high ventricular pressure $[20,21]$. 


\section{Conclusion}

In summary, with DEGs identified by gene chip data generated by high-throughput technologies from the GEO database, we constructed a cardiomyopathy double-factor regulatory network and revealed the possible mechanism on regulation level. A subnetwork under the regulation of $S R F$ and $M E F 2 C$ was also constructed to illustrate their possible regulatory roles. Our results may facilitate further therapeutic studies of cardiomyopathy.

\section{Abbreviations}

DEG: differentially expressed genes; GEO: Gene Expression Omnibus; IPA: Ingenuity Pathway Analysis; miRNAs: microRNAs; RMA: Robust Multi-array Analysis; TF: transcription factor; UCSC: University of California Santa Cruz.

\section{Competing interests}

The authors declare that they have no competing interests.

\section{Authors' contributions}

LW conceived of the study and drafted the manuscript. JH, HX and MS performed the statistical analysis. JW, QJ and HY revised the manuscript. All authors read and approved the final manuscript.

\section{Acknowledgement}

This study is supported by Provincial Natural Science Basic Research Foundation of Shaanxi (2014JM4152) and the Science and Technology Project of Xi'an Municipal Health Bureau (Number 2013028).

Received: 31 July 2014 Accepted: 14 October 2014

Published online: 24 October 2014

\section{References}

1. Maron BJ, Towbin JA, Thiene G, Antzelevitch C, Corrado D, Arnett D, Moss AJ, Seidman CE, Young JB, American Heart Association; Council on Clinical Cardiology, Heart Failure and Transplantation Committee; Quality of Care and Outcomes Research and Functional Genomics and Translational Biology Interdisciplinary Working Groups; Council on Epidemiology and Prevention: Contemporary definitions and classification of the cardiomyopathies: an American Heart Association scientific statement from the council on clinical cardiology, heart failure and transplantation committee; quality of care and outcomes research and functional genomics and translational biology interdisciplinary working groups; and council on epidemiology and prevention. Circulation 2006, 113:1807-1816.

2. Towbin JA, Lowe AM, Colan SD, Sleeper LA, Orav EJ, Clunie S, Messere J, Cox GF, Lurie PR, Hsu D, Canter C, Wilkinson JD, Lipshultz SE: Incidence, causes, and outcomes of dilated cardiomyopathy in children. JAMA 2006, 296:1867-1876.

3. Taylor MR, Carniel E, Mestroni L: Cardiomyopathy, familial dilated. Orphanet J Rxare Dis 2006, 1:27.

4. Ferreira LR, Frade AF, Santos RH, Teixeira PC, Baron MA, Navarro IC, Benvenuti LA, Fiorelli Al, Bocchi EA, Stolf NA, Chevillard C, Kalil J, CunhaNeto E: MicroRNAs miR-1, miR-133a, miR-133b, miR-208a and miR-208b are dysregulated in chronic Chagas disease cardiomyopathy. Int J Cardiol 2014, 175:409-417.

5. Xu HF, Ding YJ, Zhang ZX, Wang ZF, Luo CL, Li BX, Shen YW, Tao LY, Zhao ZQ: MicroRNA21 regulation of the progression of viral myocarditis to dilated cardiomyopathy. Mol Med Reports 2014, 10:161-168.

6. Hannenhalli S, Putt ME, Gilmore JM, Wang J, Parmacek MS, Epstein JA, Morrisey EE, Margulies KB, Cappola TP: Transcriptional genomics associates FOX transcription factors with human heart failure. Circulation 2006 114:1269-1276.

7. Irizarry RA, Hobbs B, Collin F, Beazer-Barclay YD, Antonellis KJ, Scherf U, Speed TP: Exploration, normalization, and summaries of high density oligonucleotide array probe level data. Biostatistics 2003, 4:249-264.

8. Benjamini $Y$, Hochberg $Y$ : Controlling the false discovery rate: a practical and powerful approach to multiple testing. J R Stat Soc Ser B Methodol 1995, 57:289-300.
9. Grimson A, Farh KK, Johnston WK, Garrett-Engele P, Lim LP, Bartel DP: MicroRNA targeting specificity in mammals: determinants beyond seed pairing. Mol Cell 2007, 27:91-105

10. John B, Enright AJ, Aravin A, Tuschl T, Sander C, Marks DS: Human MicroRNA targets. PLOS Biol 2004, 2:e363.

11. Kertesz M, lovino N, Unnerstall U, Gaul U, Segal E: The role of site accessibility in microRNA target recognition. Nat Genet 2007, 39:1278-1284.

12. Rickard AJ, Funder JW, Morgan J, Fuller PJ, Young MJ: Does glucocorticoid receptor blockade exacerbate tissue damage after mineralocorticoid/salt administration? Endocrinology 2007, 148:4829-4835.

13. Tokudome S, Sano M, Shinmura K, Matsuhashi T, Morizane S, Moriyama H, Tamaki K, Hayashida K, Nakanishi H, Yoshikawa N, Shimizu N, Endo J, Katayama T, Murata M, Yuasa S, Kaneda R, Tomita K, Eguchi N, Urade Y, Asano K, Utsunomiya Y, Suzuki T, Taguchi R, Tanaka H, Fukuda K: Glucocorticoid protects rodent hearts from ischemia/reperfusion injury by activating lipocalin-type prostaglandin D synthase-derived PGD2 biosynthesis. J Clin Invest 2009, 119:1477-1488.

14. Alonso-Montes C, Naves-Diaz M, Fernandez-Martin JL, Rodriguez-Reguero J, Moris C, Coto E, Cannata-Andia JB, Rodriguez I: New polymorphisms in human MEF2C gene as potential modifier of hypertrophic cardiomyopathy. Mol Biol Rep 2012, 39:8777-8785.

15. Xu J, Gong NL, Bodi I, Aronow BJ, Backx PH, Molkentin JD: Myocyte enhancer factors $2 \mathrm{~A}$ and $2 \mathrm{C}$ induce dilated cardiomyopathy in transgenic mice. J Biol Chem 2006, 281:9152-9162.

16. Dasqupta T, Stillwagon SJ, Ladd AN: Gene expression analyses implicate an alternative splicing program in regulating contractile gene expression and serum response factor activity in mice. PLoS One 2013, 8:e56590.

17. Tritsch E, Mallat Y, Lefebvre F, Diguet N, Escoubet B, Blanc J, De Windt LJ, Catalucci D, Vandecasteele G, Li Z, Mericskay M: An SRF/miR-1 axis regulates $\mathrm{NCX} 1$ and annexin $\mathrm{A} 5$ protein levels in the normal and failing heart. Cardiovasc Res 2013, 98:372-380.

18. Jiang J, Wakimoto H, Seidman JG, Seidman CE: Allele-specific silencing of mutant Myh6 transcripts in mice suppresses hypertrophic cardiomyopathy. Science 2013, 342:111-114.

19. Lu D, Zhang L, Bao D, Lu Y, Zhang X, Liu N, Ge W, Gao X, Li H, Zhang L: Calponin 1 inhibits dilated cardiomyopathy development in mice through the epsilonPKC pathway. Int J Cardiol 2014, 173:146-153.

20. Asanuma H, Kitakaze M: Carperitide and adiponectin - how are they connected each other to benefit acute decompensated heart failure? Circ J 2009, 73:2206-2207. http://www.ncbi.n/m.nih.gov/pubmed/19940369.

21. Tarazon E, Rosello-Lleti E, Rivera M, Ortega A, Molina-Navarro MM, Trivino JC, Lago F, Gonzalez-Juanatey JR, Orosa P, Montero JA, Salvador A, Portolés $M$ : RNA sequencing analysis and atrial natriuretic peptide production in patients with dilated and ischemic cardiomyopathy. PLoS One 2014, 9:e90157.

doi:10.1186/s40001-014-0057-5

Cite this article as: Wang et al:: Construction of microRNA and transcription factor regulatory network based on gene expression data in cardiomyopathy. European Journal of Medical Research 2014 19:57.

\section{Submit your next manuscript to BioMed Central and take full advantage of:}

- Convenient online submission

- Thorough peer review

- No space constraints or color figure charges

- Immediate publication on acceptance

- Inclusion in PubMed, CAS, Scopus and Google Scholar

- Research which is freely available for redistribution 